\title{
XEN Gel Implant
}

\author{
Leon Au and Ingeborg Stalmans
}

\subsection{Device Design and Evolution}

The concept of the XEN Glaucoma Treatment System was initially developed at the Lions Eye Institute in Perth, Australia. It was commercialized by the company AqueSys, Inc. (Fort Worth, Texas, USA) which later was acquired by Allergan plc (Dublin, Ireland) in 2015. The concept was to create a subconjunctival aqueous drainage pathway similar to trabeculectomy, but via an ab-interno approach. The XEN Gel Implant is a 6-mm hydrophilic collagen cylindrical implant comprising of cross-linked porcine gelatin. It has an external diameter of $150 \mu \mathrm{m}$ and an internal lumen of $45 \mu \mathrm{m}$. It aims to provide a direct permanent communication between the anterior chamber and the subconjunctival space (Fig. 6.1). It is rigid when dry, but softens and swells externally after immersion in aqueous. The soft gelatinous property was thought to improve biocompatibility in the subconjunctival space and reduce the risk of erosion, while the small amount of external expansion aids device anchorage and minimizes migration. The XEN Glaucoma Treatment System received the CE mark in 2013 and was approved by the FDA in 2016. The lumen size of the XEN Gel Implant has changed from the initial $140 \mu \mathrm{m}$ and later, $63 \mu \mathrm{m}$ diameter versions, to the current, commercially available, $45 \mu \mathrm{m}$ (also known as the $\mathrm{XEN}-45$ implant), which is claimed to provide approximately $6-8 \mathrm{mmHg}$ internal pressure resistance according to the Hagen-Poiseuille law and protect against postoperative hypotony [1].

\footnotetext{
L. Au $(\bowtie)$

Manchester Royal Eye Hospital, Manchester University Foundation Trust, Manchester, UK Medical Academic Health Sciences Centre, University of Manchester, Manchester, UK

I. Stalmans

Department of Ophthalmology, University Hospitals Leuven, Leuven, Belgium

Department of Neurosciences, Research Group of Ophthalmology, KU Leuven, Leuven, Belgium 

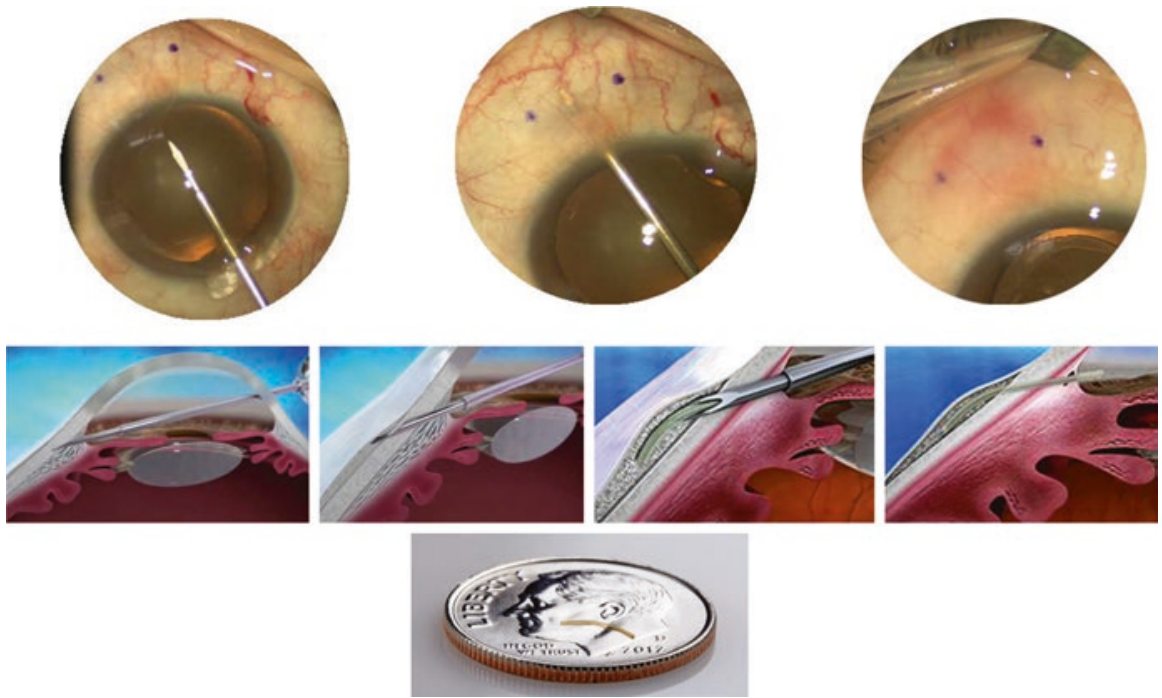

Fig. 6.1 The XEN Glaucoma Treatment System. (Copyright Allergan plc, Dublin, Ireland; reproduced with permission)
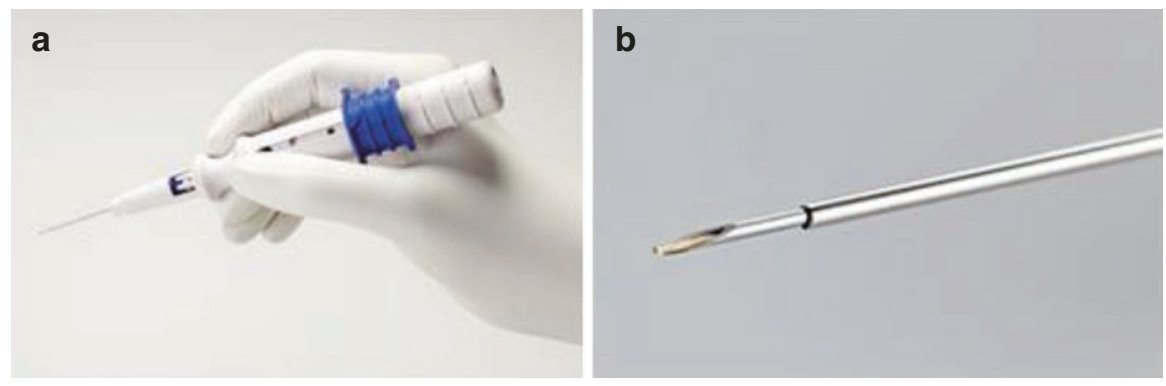

Fig. 6.2 The XEN Injector. (a) The XEN Gel Implant is preloaded in a disposable single-use injector. When the blue slider is moved forward, the XEN Gel Implant is injected into the subconjunctival space. (b) The tip of the injector comprises of a 27 -gauge double beveled needle. (Copyright Allergan plc, Dublin, Ireland; reproduced with permission)

The XEN Gel Implant is preloaded in the XEN injector that is sterile and for single use only (Fig. 6.2). The injector comprises a straight 27 -gauge double beveled needle with the XEN Gel Implant preloaded, a white surgical handle and a blue slider that deploys the implant. The injector advances across the anterior chamber through an inferotemporal clear corneal incision and delivers the XEN Gel Implant into the superonasal quadrant of the subconjunctival or sub-Tenon's space (Fig. 6.3). On completion of the deployment, the XEN Gel Implant should be placed $1 \mathrm{~mm}$ in the anterior chamber and $2 \mathrm{~mm}$ within the sclera, leaving a 3-mm extraocular portion under the conjunctiva. The implant should exit the sclera 3-mm posterior to the limbus, ideally creating a posterior filtration bleb. This is augmented by the use of 
Fig. 6.3 Supero-nasal XEN Gel Implant associated with a diffuse bleb. (Copyright Leon Au, FRCOphth; reproduced with permission)

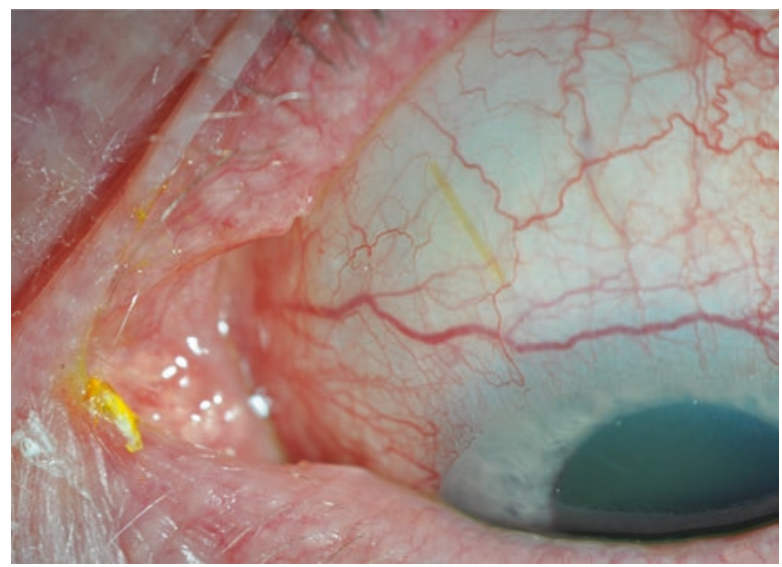

the antimetabolite, Mitomycin-C (MMC), which is typically given as a subconjunctival injection prior to device implantation.

\subsection{Patient Selection}

According to the CE mark, the XEN Gel Implant is intended to reduce intraocular pressure (IOP) in patients with primary open-angle glaucoma where previous medical treatments have failed. The Xen Glaucoma Treatment System was approved in the United States for the management of refractory glaucoma, where previous surgical treatment has failed, or in patients with primary open-angle glaucoma, pseudoexfoliative or pigmentary glaucoma that is unresponsive to maximum tolerated medical therapy.

This procedure is suitable for patients with an open drainage angle, typically Shaffer grade 3 or above. The main advantages of the XEN Gel Implant over other filtering procedures include its less invasive surgical procedure which does not require conjunctival peritomy, the favorable safety profile, fast visual recovery, and short surgical duration, rendering this implant especially appropriate for patients who are unable to tolerate long surgical procedures or those who cannot accept prolonged visual recovery. Although designed as a stand-alone procedure, XEN implantation can be combined with phacoemulsification in patients with concurrent cataract. Since the implant is placed in the superonasal quadrant, other surgical options involving the supero-temporal quadrant are still an option in case of filtration failure.

The outcome after XEN implantation is dependent on the formation and maintenance of a filtering bleb. In contrast to conventional trabeculectomy where the outflow can be manipulated postoperatively via suture removal, the XEN Gel Implant is a fixed-flow device. As a result, its success is greatly dependent on the postoperative subconjunctival resistance. Therefore, patients at risk of bleb fibrosis are likely to have a less favorable surgical outcome, and careful patient selection is crucial for achieving success with this procedure. Known risk factors for fibrosis after filtering 
Fig. 6.4 A patient with deep and sunken superior sulcus which could result in difficulties in access during XEN surgery. (Copyright Ingeborg Stalmans, MD; reproduced with permission)

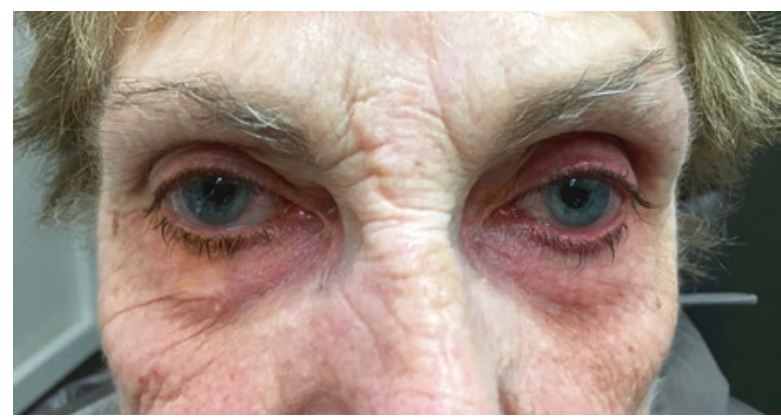

surgery include younger age, darker skin color, multiple topical glaucoma medications, diabetes, systemic autoimmune diseases, and a history of previous ocular surgery, especially procedures involving the conjunctiva. Moreover, preexisting intraocular and ocular surface inflammation increases the risk of postoperative fibrosis. Therefore, careful preoperative slit-lamp examination, including assessment of the inflammatory status, integrity, and mobility of the (superonasal) conjunctiva, as well as goniocopy to assess the angle, is mandatory for appropriate patient selection. Preoperative ocular surface disease and inflammation should be treated for ideally at least 1 month prior to surgery. Surgeons can consider altering or reducing the topical glaucoma medications before the surgery to reduce inflammation and decrease the preservative load, prescribing topical steroids or even switching from topical glaucoma medications to oral acetazolamide if there are no contraindications. Lid disease, blepharitis, and chronic dry eyes should be treated appropriately before the surgery.

It is important to achieve good surgical exposure for this procedure, especially because the procedure is performed with the eye in the primary position, as compared to conventional filtration surgery in which a corneal traction suture is used to rotate the eye downward. Hence patients need to be assessed preoperatively to ascertain whether the palpebral aperture is sufficiently wide and to identify factors which may cause surgical difficulties, including tight eyelids (e.g., history of contact dermatitis), a deep sunken sulcus or high cheekbones (Fig. 6.4). A comprehensive medical and surgical history should also be documented. Uncontrolled systemic hypertension or the use of oral anticoagulants for valvular heart disease increases the risk of intraocular and subconjunctival hemorrhage, the latter of which contributes to bleb fibrosis and surgical failure. Lastly, MMC is required for this procedure; hence, limbal stem cell failure and pregnancy are contraindications.

\subsection{Surgical Technique}

To indicate the intended implantation site, the conjunctiva is marked $3 \mathrm{~mm}$ from the limbus in the superonasal quadrant, close to the 12 o'clock position. Hydroexpansion of the tenon's capsule is performed by injecting MMC subconjunctivally in the target quadrant, at least $5 \mathrm{~mm}$ posterior to the limbus. An inferotemporal clear 
corneal incision is made at approximately $1 \mathrm{~mm}$ anterior to the limbus and an additional small incision is made nasally at the limbus. The anterior chamber is filled completely with a cohesive viscoelastic. The preloaded Xen injector is introduced across the anterior chamber through the main incision. The needle tip is aimed at the superonasal angle on the opposite side, ideally anterior to the pigmented trabecular meshwork and Schlemm's canal. Intraoperative gonioscopy can be used to position the injector needle precisely, before perforating the sclera to enter the subconjunctival space $2.5-3 \mathrm{~mm}$ behind the limbus. After ensuring that the entire bevel of the needle has exited the sclera and is within the subconjunctival space, the slider on the injector is moved forward. During the first half of the slider movement, the distal part of the implant is ejected from the needle tip into the subconjunctival space. Further movement of the slider forward results in the retraction of the needle into the injector while releasing the remainder of the implant in the sclera and anterior chamber. After the needle has fully retracted into the injector, it is removed from the anterior chamber. The position and the mobility of the subconjunctival segment of the implant are assessed and the correct length and position of the internal segment are confirmed by gonioscopy. Viscoelastic is removed from the anterior chamber and the incisions are hydrated (suturing is optional). The anterior chamber is irrigated and slightly pressurized to ensure that a filtration bleb is formed.

When XEN implantation is combined with phacoemulsification, cataract surgery is usually performed first. At the end of the cataract surgery, the anterior chamber is refilled with cohesive viscoelastic. XEN implantation can be performed through the main corneal incision used for phacoemulsification (if temporal) or an additional corneal incision can be made. The subsequent steps are similar to the solo procedure.

\subsubsection{Avoiding Complications and Surgical Pearls}

This section provides a step-by-step approach to the surgical procedure details, with practical recommendations on how to refine the surgical technique and to avoid and correct implant placement imperfections.

The surgeon can be seated superiorly or temporally. It is recommended that surgeons starting off with XEN implantation sit in their normal position for phacoemulsification, but also, as they gain confidence, try the alternative position. Similarly, there are several ways to hold the injector. The most comfortable hand position is also surgeon-dependent and is determined by a number of factors such as the hand size, the position of the surgeon relative to the patient, etc. However, holding the injector in the right hand when operating on a right eye and the left hand when operating on a left eye is advisable. Surgeons in training should try different seating and hand positions in the dry lab first, in order to find the most comfortable configuration. For the initial few cases, one should consider implanting pseudophakic patient with good surgical exposure and the right eye for the right-handed surgeon and vice versa. One should avoid implanting phakic patients until experienced with the technique.

The choice of anesthesia is also at the discretion of the surgeon. The majority of the procedures are performed under topical anesthesia, using anesthetic drops in 
combination with an intracameral supplement (separately injected in the anterior chamber or as a combo-product with the viscoelastic) and/or subconjunctival anesthesia (either in combination with the hydro-expansive fluid or separately). When used subconjunctivally, the anesthetic can be complemented with adrenaline as a vasoconstrictor. Some surgeons prefer a sub-tenon, peribulbar, or retrobulbar anesthesia. The advantage of the latter is deeper anesthesia, ensuring a painless procedure. In addition, for the novice, these provide akinesia, making the procedure easier. On the other hand, hydro-expansion may be more difficult because the patient cannot be instructed to look down to expose the posterior superior bulbar conjunctiva. We would encourage surgeons to move toward topical anesthesia when their techniques mature, because of the advantage of better access especially for hydro-expansion of the conjunctiva and delivery of MMC posteriorly into the fornix rather than at the limbus (Fig. 6.5).

To avoid excessive sub-conjunctival hemorrhage during hydro-expansion (which may obscure the implantation site and make the implantation procedure more challenging), a vasoconstrictor, such as topical apraclonidine (Iopidine ${ }^{\circledR}$, Alcon, Fort Worth, Texas, USA) may be administered immediately prior to the surgery. This can counteract the hyperemia which is often caused by chronic glaucoma medication or pilocarpine administered preoperatively to constrict the pupil and protect the lens during standalone procedures. Alternatively, one can add adrenaline to the hydroexpansion fluid, as mentioned above or apply topical adrenaline. This has the potential disadvantage of pupil dilation, which can be prevented by preoperative topical pilocarpine. Ideally, one should use a fine (30G) needle for the hydro-expansion and carefully place the needle to avoid perforating conjunctival blood vessels. If subconjunctival hemorrhage does occur, one can immediately inject some fluid at the intended implantation site to prevent the blood from spreading to that area or compress the conjunctiva with a cotton tip and massage the blood away.

The use of antimetabolites in the hydro-expansion fluid is highly recommended. Although off-label, MMC is widely used in filtering surgery and accepted as common practice to prevent scarring and improve surgical outcomes. The concentration of the MMC can be titrated according to the patient profile which determines the anticipated scarring tendency (e.g., age, race, previous surgical history, etc.). The

Fig. 6.5 Posterior injection of mitomycin-C used for intra-tenon's hydroexpansion. (Copyright Ingeborg Stalmans, MD; reproduced with permission)

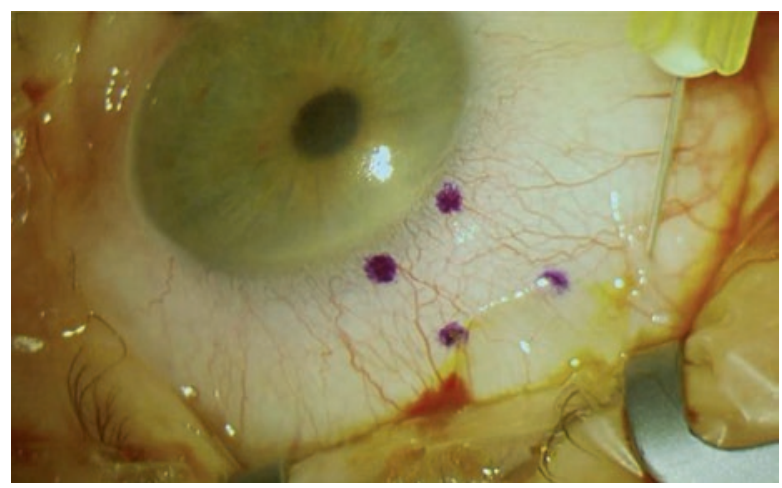


dose typically used is $0.02 \mathrm{mg}$ in a volume of $0.1 \mathrm{~mL}$, although it can vary between 0.01 and $0.05 \mathrm{mg}$. It is important to stress that the usage of MMC dosage greater than $0.02 \mathrm{mg}$ is uncommon with the Xen and should be seen as exception rather than the rule, because of the higher risk of bleb avascularity and associated complications. To avoid an avascular bleb at the limbus and the risk of postoperative blebitis or endophthalmitis in rare cases [2, 3], MMC is injected as far posteriorly as possible and massaged backward with a cotton tip if it spreads close to the limbus.

After hydro-expansion, the fluid ideally lifts the conjunctiva slightly at the intended implantation site, to reduce the risk of conjunctival perforation with the injector needle as it comes out of the sclera, without obscuring the implantation site due to excessive chemosis.

The inferotemporal incision is made $1 \mathrm{~mm}$ from the limbus in order to approach the implantation site at the appropriate angle. In a patient with a prominent cheekbone, the surgeon may want to rotate the eye slightly between the injector and the side instrument, such that the injector is rotated away from the patient's cheek to a more temporal position. Alternatively, a more temporal incision may be considered, in combination with a more tangential approach to the superonasal angle rather than crossing the pupil axis (Fig. 6.6). A gentle face turn toward the temporal direction would also help to lower the cheekbone while the eye is brought slightly nasal to maintain a primary gaze during implantation. A nasal placement of the implant, however, is not recommended because of a higher incidence of dysesthesia from nasally placed blebs and potentially greater risk of implant erosion through the conjunctiva in nasally placed implants (presumably because of rubbing of the eyelid over the implant) (Fig. 6.7).

A cohesive viscoelastic material is used to fill the anterior chamber, providing a stable anterior chamber during implantation. This can also be removed more easily and more completely than a dispersive viscoelastic. We would recommend filling up the anterior chamber firmly during implantation, as it is more difficult to pierce

Fig. 6.6 Mild cyclorotation of the eye during XEN Gel Implant surgery, associated with a tangential approach of the injector which does not cross the pupil axis, so as to facilitate access to the superior-nasal quadrant of the eye in a patient with prominent cheekbones. (Copyright Leon Au, FRCOphth; reproduced with permission)

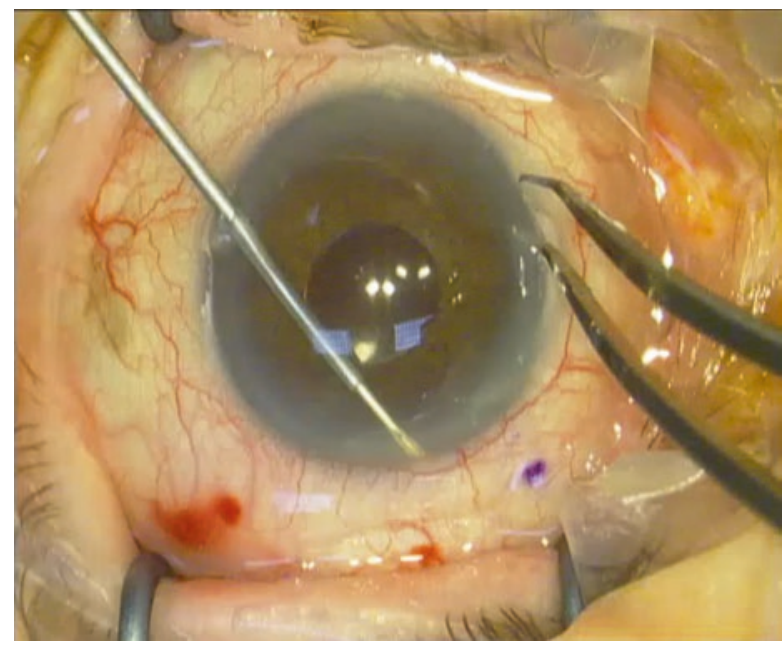



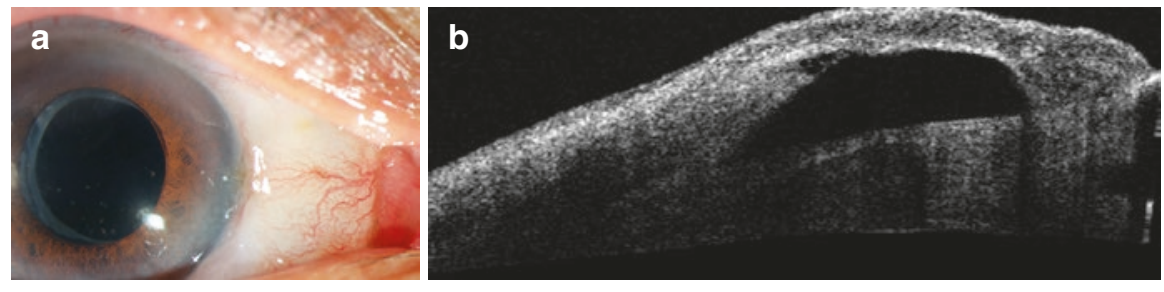

Fig. 6.7 Nasal placement of the XEN Gel Implant could result in a large nasal bleb. (a) A large nasal bleb which results in significant bleb dysesthesia. (b) Anterior segment optical coherence tomography image of the nasal bleb in (a), showing a large bleb cavity. (Copyright Chelvin Sng, FRCSEd; reproduced with permission)

sclera with the injector needle when the eye is soft. Similarly, avoid an excessively large corneal incision which allows viscoelastic to escape. The incision site needs to be marginally larger than 20 gauge to accommodate the injector. While small phaco wounds may be satisfactory, larger wounds can result in significant viscoelastic loss. A combined viscoelastic with anesthetic may also be used to complement the topical anesthesia and prevent pain during the procedure.

While entering the eye with the injector through the main port, small sidewise movements may facilitate smooth entry through the incision with the needle. A side instrument is used to stabilize the eye during the needle placement and injection. Various instruments can be used for this purpose, e.g., the Vera hook (Katena, USA), an iris spatula or a Bonn toothed forceps to grasp the cornea in the side point. As mentioned above, it is advisable to place the implant close to the 12 o'clock position, as nasal placement has been associated with bleb dysesthesia. Ideally, the implant is inserted between the pigmented trabeculum and Schwalbe's line. More posterior placement is associated with a higher risk of blood reflux from Schlemm's canal and ostium occlusion by the iris. More anterior placement can result in a very short intrascleral portion, instability of the implant and, occasionally, intracorneal placement. During the learning curve, it may be advisable to use an intraoperative indirect gonioscope to guide the injector needle while approaching the angle. However, with practice, the use of the gonioscope at this stage could later be omitted.

As the needle traverses the sclera, some forward pressure is required and the side instrument can serve to exert counterpressure. To reduce scleral resistance, and hence the amount of forward pressure on the injector required for placement, the injector can be gently rolled back and forth between the fingers creating a rotational movement of the needle. The needle should exit the sclera at $2.5-3 \mathrm{~mm}$ from the limbus. If necessary, the inserter can be tilted up- or downwards slightly during the insertion process to make the intrascleral portion longer or shorter. A longer intrascleral portion can reduce para-implant leakage and subsequent early hypotony, which may be more frequent in highly myopic eyes or those with thin sclera. A slow and controlled exit from the sclera is important to avoid puncturing the conjunctiva. There is a continuous debate about the ideal position of the implant in relation to the conjunctiva/tenon layers. Some surgeons prefer to aim the injector needle upwards, placing the implant in the superficial layers of the subconjunctival space, and increasing the chance of a freely mobile implant with easier drainage. In an ideal 
world, one would have the implant exit in the sub-Tenon's space behind Tenons insertion to avoid a thin bleb and implant erosion and maximize the use of the potential space available. The counterargument is that visualization of the patency of the XEN in the sub-Tenon's space is more difficult. Once the needle is fully advanced and the bevel has emerged completely from the sclera, the injector should be rotated $90^{\circ}$ with the bevel facing the 12 o'clock position, before advancing the slider. During the first half of this manoeuver, the implant is ejected and during the second half, the needle is retracted. When the slider reaches the point of transition, a slight resistance can be felt in the finger moving the slider. At that point, one should pause, zoom out to get an overview of the cornea, and relax the hands to release any tension on either the injector or the side instrument. This is extremely important to avoid the so-called flicks, which are caused by tension on the injector during the retraction phase. At the point where the needle is retracted into the injector, the tip is released from the angle disengaging the anchorage. Any tension at that time can result in a flicking movement of the injector to one side or the other. This can drag the implant back into the anterior chamber causing an excessively long intraocular portion. The sudden movement can also result in a hemorrhage, an enlargement of the implantation canal or, in the case of a downward flick, iris trauma, and even cyclodialysis cleft formation in extreme cases.

When the injector is being retracted, the attention of the surgeon should therefore shift from the subconjunctival needle tip to the corneoscleral limbus. One should keep a forward bias on the injector to ensure constant contact between the injector and the angle. Only when the slider has reached the forward end of its travel (and therefore the needle is completely retracted), can the injector be removed safely from the anterior chamber. Premature removal can result in damage to the angle or dislocation of the implant. Common causes of suboptimal implant placement, most commonly implants that are too long in the anterior chamber, are incomplete needle advancement into the subconjunctival or sub-Tenon's space, incomplete slider advancement, early injector retraction, and flicks.

After implantation, it is important to check the position and mobility of the implant by gently moving the implant sidewise in both directions using a blunt instrument. Ideally, the implant should be $1 \mathrm{~mm}$ in the anterior chamber, $2 \mathrm{~mm}$ in the sclera, and $3 \mathrm{~mm}$ in the subconjunctival space (the so-called 1-2-3 configuration, see Fig. 6.8). The subconjunctival part of the implant should be straight and freely mobile sidewise. If the implant is not freely mobile or is curled, because it is stuck in Tenon's capsule (Fig. 6.9), the risk of occlusion by Tenon's, postoperative fibrosis and bleb failure is higher, even if the implant functions initially. Many surgeons will perform a primary needling in this situation. A $30 \mathrm{G}$ needle is inserted under the conjunctiva at a distance from the implant, and the tip of the implant is approached carefully avoiding the blood vessels. The Tenon's capsule is moved away from the implant by gently swiping over and under the implant with the needle, paying attention not to cut or pull out the implant. This maneuver should result in a straight and mobile implant. An alternative is to gently tease the implant using a pair of tying forceps. It is sometimes possible to free an implant from Tenon's by this method.

If no bleb is visible after removal of the viscoelastic, the internal position of the implant should be checked by performing gonioscopy. The ideal position of the 

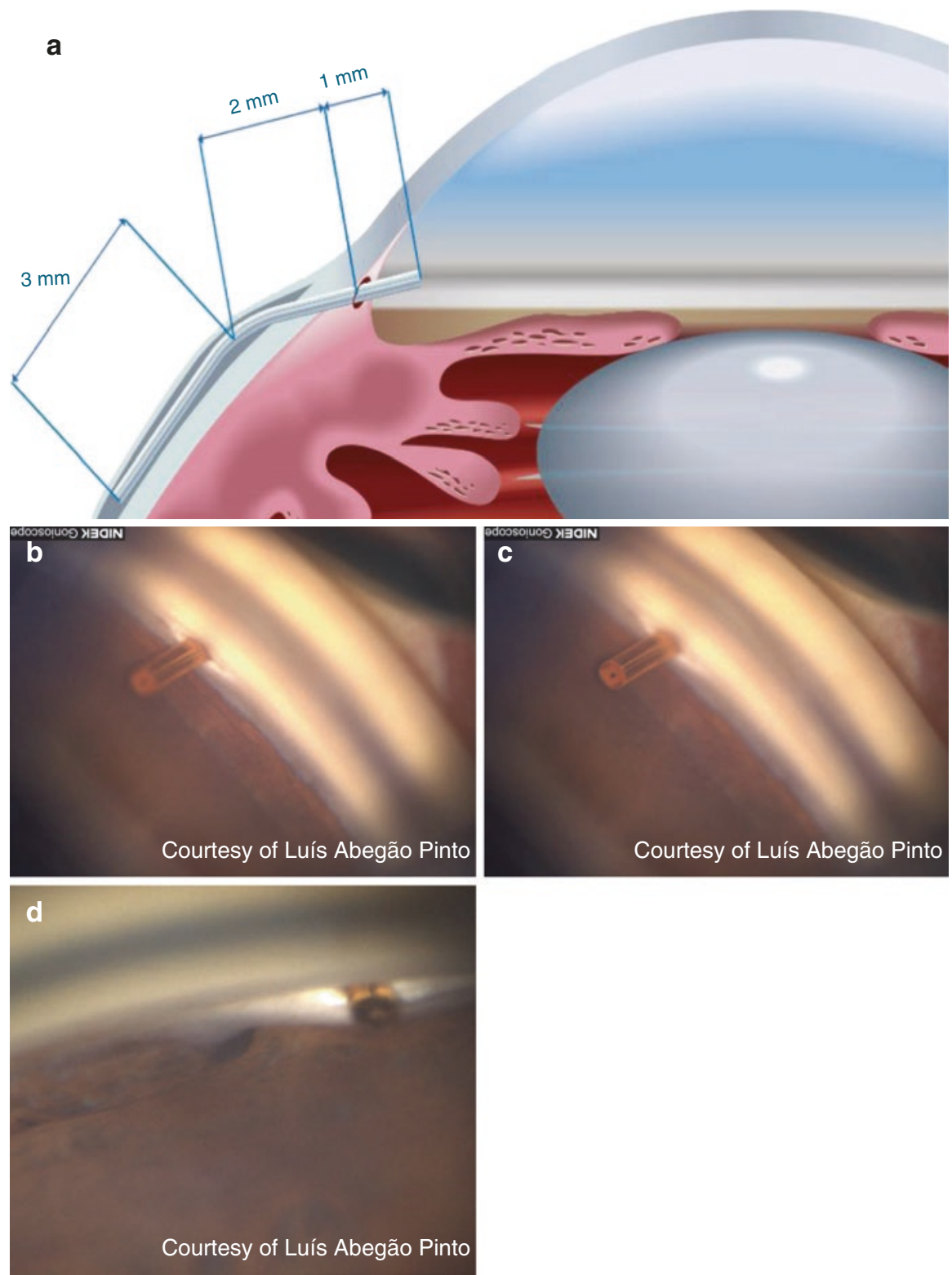

Fig. 6.8 The 1-2-3 configuration of the XEN Gel Implant. (a) The ideal placement of the XEN Gel Implant is $1 \mathrm{~mm}$ in the anterior chamber, $2 \mathrm{~mm}$ in the sclera, and $3 \mathrm{~mm}$ in the subconjunctival space. (Copyright Allergan plc, reproduced with permission) (b) Gonioscopic photograph focused on the entry site of the XEN Gel Implant, which is ideally between the pigmented trabeculum and the Schwalbe's line. (c) Gonioscopic photograph focused on the XEN Gel Implant which has an ideal intraocular segment of $1 \mathrm{~mm}$. (d) Gonioscopic photograph showing a XEN Gel Implant which has an intraocular segment which is too short. (Copyright Luís Abegão Pinto, MD; reproduced with permission) 
Fig. 6.9 A curled and immobile XEN Gel Implant which is impeded by Tenon's capsule. (Copyright Ingeborg Stalmans, MD; reproduced with permission)

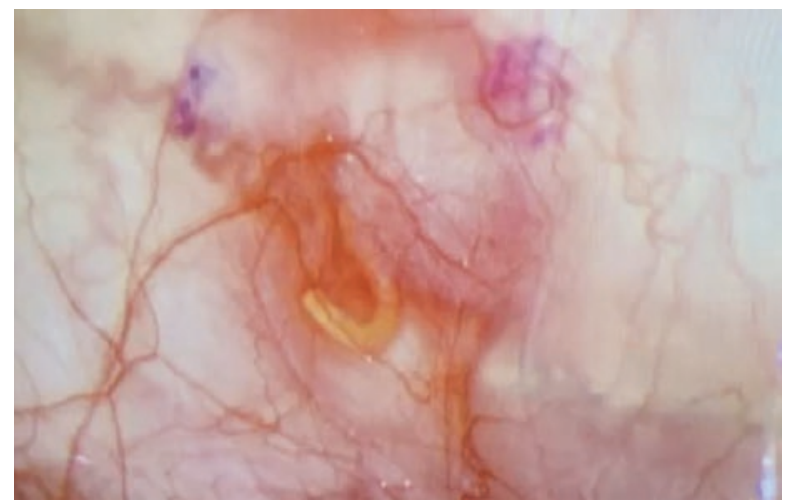

implant is between trabeculum and Schwalbe's line as mentioned previously. The iris is often pushed backward by the viscoelastic and the implant may appear free. However, if the implant is more posterior than the trabeculum, particularly if it is in contact with iris root at the scleral entry site, there is a risk of iris incarceration after viscoelastic washout. In which case, one might consider removing and reinserting the implant. An internal length of $0.5-1.5 \mathrm{~mm}$, ideally $1 \mathrm{~mm}$, is acceptable. If necessary, the length can be adjusted by gently grasping the extraocular portion of the implant through the conjunctiva with plain forceps and pushing it in or pulling it out. If the position of the implant is not satisfactory even after correction, removal, and reinsertion should be considered. To remove an implant, it may be convenient to first push the implant maximally into the anterior chamber using plain forceps e.g., Tying forceps. The implant can easily be removed from the anterior chamber using vitreoretinal forceps or aspirated using bimanual irrigation/aspiration. The injector needle is brought forward by moving the slider backward, the implant is placed in the needle again, and the insertion procedure is repeated. Care needs to be taken when handling the implant as it is now soft and can be fractured easily if excessive force is used. If there is any suspicion that the implant has been damaged, it would be more prudent to replace it with a new implant.

The final important point is the complete removal of the viscoelastic in the anterior chamber, which may otherwise block the implant in the early postoperative period and induce pressure spikes and meticulous hydration of the corneal incision. If the incisions are not watertight, they should be sutured. Thorough irrigation of the anterior chamber should result in a filtering bleb at the end of the surgery. If a filtering bleb is not visualized at the end of surgery, the implant and its position should be carefully examined and the procedure repeated if necessary. Intracameral antibiotics and subconjunctival steroids are recommended.

\subsubsection{Postoperative Management}

All pressure-lowering medications are discontinued immediately after the surgery. The typical postoperative regimen consists of a broad-spectrum topical antibiotic and intensive topical steroid (e.g., 2 hourly dexamethasone $0.1 \%$ or prednisolone 
acetate $1 \%$ ). After 2 weeks, the antibiotic eyedrop can be discontinued while topical steroids can be gradually tapered over a course of 8-10 weeks. A longer duration of topical steroid may be required in eyes with significant or persistent conjunctival hyperemia or in pigmented eyes, which are at risk of more significant conjunctival scarring.

Typically, the IOP is low over the initial postoperative days. Based on the HagenPoiseuille's law and the dimensions of the XEN Gel Implant, the IOP should theoretically be $6-8 \mathrm{mmHg}$ if the fluid passes solely through the implant without resistance in the subconjunctival space. In reality, day one IOP is often lower than $6 \mathrm{mmHg}$, presumably because the intrascleral canal produced by the injector is wider than the XEN Gel Implant, therefore allowing a small amount of para-tube leakage. In the days after the implantation, the outer diameter of the hydrophilic implant swells and its position in the sclera becomes tighter. Therefore the pressure after 1 week increases to typically around $10 \mathrm{mmHg}$.

Early hypotony usually resolves spontaneously in the first few postoperative days, requiring no additional treatment. If shallowing of the anterior chamber is a concern, a short-acting cycloplegic may be considered. Long-acting cycloplegics, such as atropine, are better avoided if the implant is posteriorly placed, because of the risk of obstructing the internal ostium of the implant with iris. In cases of significant anterior chamber shallowing or rarely when hypotony lasts 1-2 weeks after surgery, if accompanied by significant visual disturbance, corneal-lenticular touch, choroidal effusion, or maculopathy, slit-lamp injection of a small amount of dispersive visco-elastic can be used to temporize. Cohesive viscoelastics should be avoided as they may induce spikes in pressure.

A high IOP level on the first postoperative days is rare, assuming that the steps outlined above have been followed during the implantation procedure. A highpressure spike is very suggestive of a mechanical cause. Gonioscopy should be performed to confirm correct implant placement and to rule out any mechanical blockage, e.g., by iris, blood, or fibrin. The most frequent cause of an early highpressure spike is incomplete removal of the viscoelastic, in which case the pressure usually recovers in the first postoperative week. In cases of pronounced pressure spikes, anterior chamber washout should be considered. A limited hyphema generally resolves spontaneously, but if persistent or associated with an elevated pressure, a washout may rarely be required, ideally after a few days delay in order to reduce the risk of a rebleed.

If high pressure develops after the first postoperative week(s), again blockage of the internal ostium of the implant should be excluded gonioscopically. Other causes to be considered at this stage are a steroid response in combination with bleb encapsulation, especially if the bleb appears elevated. If the internal ostium is patent and there is no visible bleb elevation, then fibrosis around the implant is the more likely cause of the high pressure and needling or bleb revision may be considered. Needling is really only feasible if the implant is visible, revision can be carried out in either scenario. Encapsulation and fibrosis tend to develop after 3-4 postoperative weeks. If IOP elevation develops after 1-2 weeks, reversible mechanical obstruction of the XEN, with Tenon's rather actual fibrosis should be considered. 

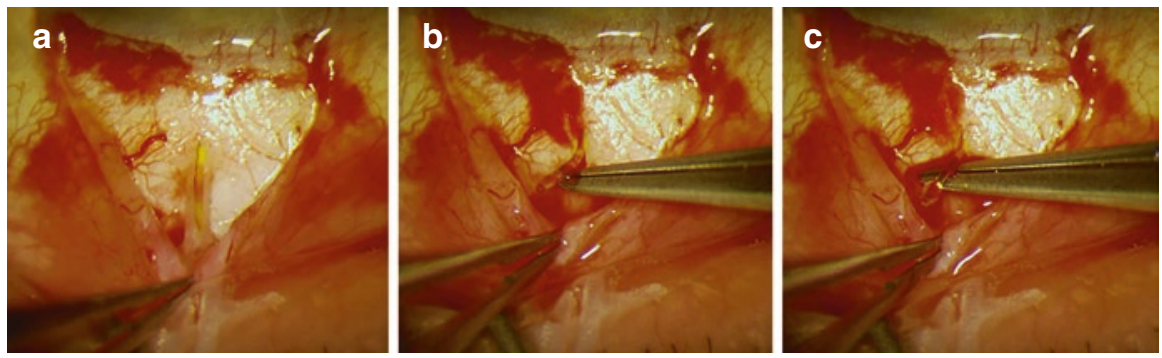

Fig. 6.10 XEN Gel Implant bleb revision. (a) A small fornix-based conjunctival peritomy is performed. (b) Subconjunctival fibrosis is carefully dissected off to expose and free up the XEN Gel Implant. (c) Once the XEN Gel Implant is mobile and free, slow aqueous flow is visible from its distal end. (Copyright Ingeborg Stalmans, MD; reproduced with permission)

Bleb revision is often performed under local anesthesia. A small fornix-based conjunctival peritomy is performed and the subconjunctival fibrosis is carefully dissected off to expose the XEN implant. The implant is often wrapped in a "sock" of tenon's tissue and a slow meticulous technique is required to free the implant without damaging it (Fig. 6.10). Once the XEN implant is mobile, one should be able to detect a slow dripping of aqueous from its distal end. Visualization may be enhanced using fluorescein drops and/or Vision Blue. If not, the implant itself might be blocked and one should consider flushing the device using a lacrimal cannula on a syringe containing BSS. If the device appears patent externally and internally but no aqueous is seen egressing from the distal end, then a fresh device should be implanted. Removal of the original non-functioning implant is unnecessary but is easy to perform at the same time. If MMC is to be used during a revision, ideally it should be applied after opening the conjunctiva but before freeing the XEN, in order to avoid any chance of MMC reflux into the anterior chamber. After the revision is complete, conjunctiva is closed in a similar fashion as conventional trabeculectomy. The postoperative eyedrop regime is similar to standard XEN Gel Implant surgery.

\subsection{Safety, Efficacy, and Clinical Results}

The APEX study group reported the 2-year results of a multicenter, prospective, nonrandomized open-label study of the XEN Gel Implant surgery in medically uncontrolled open-angle glaucoma [4]. In 202 eyes with a mean preoperative medicated IOP of $21.4 \pm 3.6 \mathrm{mmHg}$, the IOP was significantly reduced to $14.9 \pm 4.5 \mathrm{mmHg}$ at 1 year and $15.2 \pm 4.2 \mathrm{mmHg}$ at 2 years (both $p<0.001$ ). Medications were reduced from $2.7 \pm 0.9$ preoperatively to $0.9 \pm 1.1$ at 1 year and $1.1 \pm 1.2$ at 2 years. Overall, $51.1 \%$ and $47.7 \%$ of eyes were medication-free at 1 year and 2 years, respectively. There was no difference in outcome between eyes which underwent XEN implantation as a stand-alone procedure and those that underwent combined phacoemulsification with XEN implantation. Success was defined as $\geq 20 \%$ IOP reduction with the same or fewer glaucoma medications and $65.8 \%$ of eyes achieved 
that at 2 years. These findings are consistent with other published retrospective and prospective studies reporting the outcome at 1 year [5-10] (Table 6.1).

In the US pivotal trial, Grover et al. reported the 12-month outcome of XEN surgery in refractory glaucoma where $84.6 \%$ of patients had a previously failed glaucoma procedure and $56.9 \%$ required $\geq 4$ IOP lowering agents [10]. The average visual field mean deviation was $-15.0 \pm 7.7 \mathrm{~dB}$ and the mean cup-to-disk ratio was $0.82 \pm 0.13$. Despite the advanced nature of this group, $75.4 \%$ of patients achieved $\geq 20 \%$ IOP reduction with same or fewer medications at 12 months, resulting in a mean postoperative IOP of $15.9 \pm 5.2 \mathrm{mmHg}$. Nine out of the 65 eyes $(13.8 \%)$ did require a secondary glaucoma procedure during the 12 months period. It is worth noting that in this study, conjunctival peritomy was performed in all cases in order to apply the licensed MMC sponges (Mitosol, Mobius Therapeutics LLC, USA), which was different from our current implantation technique and could theoretically affect the outcome.

The postoperative needling rate of XEN surgery varies greatly. In the APEX study, the mean needling rate was $41.1 \%$ but it varied greatly between sites, ranging from $0 \%$ to $\geq 80 \%$ [4]. Over two-thirds of the needled eyes required only one episode and there was no difference between XEN alone and combined phaco-XEN surgeries. Similar needling rates were reported by others, supporting the importance of postoperative bleb management. [5-10]

The reported incidence of both intraoperative and postoperative complications was low. Numerical hypotony (IOP $<6 \mathrm{mmHg}$ ) occurred in up to $20 \%$ of patients in the initial postoperative period but mostly resolved without interventions [4-10]. Other complications, including device obstruction by iris, conjunctival erosion resulting in implant exposure, endophthalmitis, and significant visual loss, were rare.

In a retrospective study, Schlenker and colleagues evaluated the outcome of XEN surgery versus trabeculectomy in uncontrolled glaucoma with no prior incisional surgery [11]. The results demonstrated no difference in efficacy, safety, and risk of failure between the two groups, with the XEN group favoring a quicker visual recovery but a higher needling rate.

Table 6.1 Summary of published XEN studies with 1-year follow-up

\begin{tabular}{l|l|l|l|l|l|l}
\hline Authors & $\begin{array}{l}\text { No. of } \\
\text { cases }\end{array}$ & Surgery & $\begin{array}{l}\text { Preop IOP } \\
\mathrm{mmHg}\end{array}$ & $\begin{array}{l}12 \mathrm{mth} \text { IOP } \\
\mathrm{mmHg}\end{array}$ & $\begin{array}{l}\geq 20 \% \text { IOP } \\
\text { drop off Rx }\end{array}$ & $\begin{array}{l}\text { Needling } \\
(\%)\end{array}$ \\
\hline $\begin{array}{l}\text { De Gregorio } \\
\text { et al. [5] }\end{array}$ & 41 & Phaco-XEN & $22.5 \pm 3.7$ & $13.1 \pm 2.4$ & $\begin{array}{l}80.4 \% \text { (IOP } \\
6-17 \text { no } \\
\text { Rx) }\end{array}$ & 2.4 \\
\hline $\begin{array}{l}\text { Gala et al. } \\
{[6]}\end{array}$ & 13 & $\begin{array}{l}\text { XEN and } \\
\text { phaco-XEN }\end{array}$ & $16 \pm 4$ & $12 \pm 3$ & $41.7 \%$ & 30.7 \\
\hline $\begin{array}{l}\text { Hengerer } \\
\text { et al. [7] }\end{array}$ & 242 & $\begin{array}{l}\text { XEN and } \\
\text { phaco-XEN }\end{array}$ & $32.2 \pm 9.1$ & $14.2 \pm 4.0$ & $55.4 \%$ & 27.7 \\
\hline $\begin{array}{l}\text { Mansouri } \\
\text { et al. [8] }\end{array}$ & 149 & $\begin{array}{l}\text { XEN and } \\
\text { phaco-XEN }\end{array}$ & $20.0 \pm 7.1$ & $13.9 \pm 4.3$ & $57.7 \%$ & 37 \\
\hline $\begin{array}{l}\text { Tan et al. } \\
{[9]}\end{array}$ & 39 & XEN & $24.9 \pm 7.8$ & $14.5 \pm 3.4$ & $\begin{array}{l}56.2 \% \text { (IOP } \\
<18 \text { no Rx) }\end{array}$ & 51.3 \\
\hline $\begin{array}{l}\text { Grover et al. } \\
{[10]}\end{array}$ & 65 & XEN & $25.1 \pm 3.7$ & $15.9 \pm 5.2$ & $\begin{array}{l}75.4 \% \\
(s a m e ~ o r ~ \\
\text { fewer Rx) }\end{array}$ & 21 \\
\hline
\end{tabular}




\subsection{Off-Label Use}

Although the XEN Gel Implant was licensed for use in open-angle glaucoma and refractory glaucoma uncontrolled with medications or prior glaucoma surgery, its filtering property resembles that of trabeculectomy hence could potentially be adopted in other subtypes of glaucoma. Sng and colleagues reported good efficacy with the XEN Gel Implant as a treatment for uncontrolled uveitic glaucoma [2]. In their cohort of 24 consecutive patients, XEN Gel Implant surgery achieved a remarkable $60.2 \%$ IOP reduction from a high baseline IOP of $30.5 \pm 9.8 \mathrm{mmHg}$ typically found in uveitic patients; $83.3 \%$ of patients avoided further surgery at 1 year. Nevertheless, caution should be exercised when considering the XEN Gel Implant for uveitic eyes due to the significantly higher risk of postoperative hypotony. In Sng's series, $20.8 \%$ of patients required anterior chamber reformation for hypotony. In addition, the implant could be occluded by inflammatory debris in uveitic eyes or by hypotonic/atrophic iris tissue if the position is too posterior. Angle closure is currently considered a contraindication for XEN implantation. In a case series of 19 angle-closure eyes, Sng et al. reported that combined XEN implantation with cataract surgery significantly reduced the IOP $(11.7 \pm 3.0$ vs. $21.7 \pm 3.7 \mathrm{mmHg}, p<0.001)$ and the number of glaucoma medications $(0.2 \pm 0.5$ vs. $1.4 \pm 0.7, p<0.001)$ compared to baseline. The safety profile of XEN implantation in their small case series was similar to that reported for POAG eyes, though implant occlusion with iris occurred postoperatively in one angle closure eye [12]. However, they could not determine the additional effect of XEN implantation in lowering the IOP compared with phacoemulsification alone, and a randomized study comparing phacoemulsification alone with the combined procedure in angle-closure eyes is warranted. Successful XEN surgery has also been reported in patients with ICE syndrome and endothelial transplant as well as refractory glaucoma with previously failed trabeculectomy and two Ahmed valves [13,14]. D'Alessandro and colleagues reported a novel combination of the XEN Gel Implant and the Baerveldt tube in the treatment of difficult refractory glaucoma [15]. It offers the potential advantage of immediate drainage of the XEN Gel Implant and the posterior diversion of aqueous over a large plate of Baerveldt implant. The smaller XEN implant in the anterior chamber is potentially more endothelial friendly than the larger glaucoma drainage implant. However, constant friction between the XEN Gel Implant and the much large Baerveldt tube may risk longer term implant fracture at this junction, thereby disconnecting the Baerveldt from the anterior chamber. There are also concerns that the XEN may slip out of the tube unless it is sutured securely, as the external diameter of the XEN is significantly less than the internal diameter of the Baerveldt. In addition, connecting the Baerveldt tube with the XEN Gel Implant does not necessarily prevent early postoperative hypotony, which can be a consequence of peri-implant aqueous flow [16]. Notably, most of these novel applications of XEN were performed in small numbers and their longer term results are yet to be determined. 


\subsection{Conclusion}

The XEN Glaucoma Treatment System is currently the only procedure that targets the subconjunctival filtering space through an $a b$-interno approach. It offers a more significant reduction in IOP and medications than other ab-interno MIGS procedures. However, it is also arguably more technically demanding and a meticulous surgical approach is paramount. The creation of a filtering bleb requires careful pre- and postoperative management in order to secure success.

\section{References}

1. Vera VI, Horvath C. XEN gel stent: the solution designed by AqueSys. In: Samples JR, Ahmed IIK, editors. Surgical innovations in glaucoma. New York: Springer Science+Business Media; 2014. p. 189-98.

2. Sng CC, Wang J, Hau S, Htoon HM, Barton K. XEN-45 collagen implant for the treatment of uveitic glaucoma. Clin Exp Ophthalmol. 2018;46:339-45.

3. Kerr NM, Wang J, Sandhu A, Harasymowycz PJ, Barton K. Ab interno gel implant-associated bleb-related infection. Am J Ophthalmol. 2018;189:96-101.

4. Reitsamer H, Sng C, Vera V, Lenzhofer M, Barton K, Stalmans I, for the Apex study group. Two-year results of a multicenter study of the ab interno gelatin implant in medically uncontrolled open-angle glaucoma. Graefes Arch Clin Exp Ophthalmol. 2019;257:983-96.

5. De Gregorio A, Pedrotti E, Russo L, Morselli S. Minimally invasive combined glaucoma and cataract surgery: clinical results of the smallest ab interno gel stent. Int Ophthalmol. 2018;38:1129-34. https://doi.org/10.1007/s10792-017-0571-x.

6. Galal A, Bilgic A, Eltanamly R, Osman A. XEN glaucoma implant with mitomycin C 1-year follow-up: result and complications. J Ophthalmol. 2017;2017:5457246.

7. Hengerer FH, Kohnen T, Mueller M, Conrad-Hengerer I. Ab interno gel implant for the treatment of glaucoma patients with or without prior glaucoma surgery: 1-year results. J Glaucoma. 2017;26(12):1130-6.

8. Mansouri K, Guidotti J, Rao HL, et al. Prospective evaluation of standalone XEN gel implant and combined phacoemulsification-XEN Gel implant surgery: 1-year results. J Glaucoma. 2018;27(2):140-7.

9. Tan SZ, Walkden A, Au L. One-year result of XEN45 implant for glaucoma: efficacy, safety, and postoperative management. Eye. 2018;32(2):324-32.

10. Grover DS, Flynn WJ, Bashford KP, et al. Performance and safety of a new ab interno gelatin stent in refractory glaucoma at 12 months. Am J Ophthalmol. 2017;183:25-36.

11. Schlenker MB, Gulamhusein H, Conrad-Hengerer I, et al. Efficacy, safety, and risk factors for failure of standalone ab interno gelatin microstent implantation versus standalone trabeculectomy. Ophthalmology. 2017;124(11):1579-88.

12. Sng CCA, Chew PTK, Htoon HM, et al. Case series of combined XEN implantation and phacoemulsification in Chinese eyes: one-year outcomes. Adv Ther. 2019;36(12):3519-29.

13. Sandhu S, Dorey MW. Case report: Xen Ab Interno gel stent use in a refractory glaucoma patient with previous filtration surgeries. J Glaucoma. 2018;27(3):e59-60.

14. Hohberger B, Welge-Lüen UC, Lämmer R. ICE-syndrome: a case report of implantation of a microbypass Xen gel stent after DMEK transplantation. J Glaucoma. 2017;26(2):e103-4.

15. D'Alessandro E, Guidotti JM, Mansouri K, Mermoud A. XEN-augmented Baerveldt: a new surgical technique for refractory glaucoma. J Glaucoma. 2017;26(2):e90-2.

16. Sng CCA, Wang J, Barton K. Caution in using the XEN-augmented Baerveldt surgical technique. J Glaucoma. 2017;26:e257. 
Open Access This chapter is licensed under the terms of the Creative Commons Attribution 4.0 International License (http://creativecommons.org/licenses/by/4.0/), which permits use, sharing, adaptation, distribution and reproduction in any medium or format, as long as you give appropriate credit to the original author(s) and the source, provide a link to the Creative Commons license and indicate if changes were made.

The images or other third party material in this chapter are included in the chapter's Creative Commons license, unless indicated otherwise in a credit line to the material. If material is not included in the chapter's Creative Commons license and your intended use is not permitted by statutory regulation or exceeds the permitted use, you will need to obtain permission directly from the copyright holder. 